\title{
Percutaneous C-arm-guided Wide Bore Needle Biopsy for Intraosseous Spinal Lesions
}

\author{
${ }^{1}$ Saumyajit Basu, ${ }^{2}$ Agnivesh Tikoo, ${ }^{3}$ Farid H Malik, ${ }^{4}$ Jay Deep Ghosh, ${ }^{5}$ Mantu Jain, ${ }^{6}$ Trinanjan Sarangi
}

\section{ABSTRACT}

Study design: Retrospective analysis of data of those patients who underwent a percutaneous transpedicular biopsy at our hospital was done. All patients had a bony lesion in a vertebra (thoracic, lumbar, sacrum) without a soft tissue component around the bone and neurodeficit.

Objective: To analyze the role of percutaneous wide bore needle biopsy in vertebral lesions without any soft tissue component.

Summary of background data: Adequate treatment of spinal lesions requires formulation of diagnosis-best achieved by a tissue biopsy when all attempts at diagnosis fail by noninvasive methods. Percutaneous CT guided fine needle biopsy is technically difficult in intraosseous lesions leading to frequent inconclusive results and hence the necessity of wide bore needle biopsy.

Materials and methods: Retrospective analysis of data of 26 patients with mean age of 58.8 years who underwent a percutaneous transpedicular biopsy at our hospital was done. All patients had a bony lesion in a vertebra (thoracic, lumbar, saccrum) without a soft tissue component around the bone and neurodeficit. The procedure was done under local anesthesia with sedation.

Results: Positive diagnosis was achieved in 23 out of 26 , i.e. $88.4 \%$ of cases (adequacy). Out of 26 , there were 13 cases of malignancy (50\%), 8 cases of tuberculosis $(30.7 \%)$, 2 cases of osteoporosis (7.6\%) and biopsy was inconclusive in $3(11.5 \%)$ cases. Of the 13 malignancies, 7 cases were of metastasis $(53.8 \%), 5$ cases of plasmocytoma $(38.4 \%)$ and 1 case of lymphoma (7.6\%).

Conclusion: Percutaneous biopsy under fluoroscopic guidance by transpedicular approach is quite safe and gives high adequacy $(88.4 \%)$ without significant complications that are associated with open and paraspinal techniques.

Keywords: Spinal biopsy, Percutaneous biopsy, Vertebral metastasis, Wide bore biopsy.

How to cite this article: Basu S, Tikoo A, Malik FH, Ghosh JD, Jain M, Sarangi T. Percutaneous C-arm-guided Wide Bore Needle Biopsy for Intraosseous Spinal Lesions. J Postgrad Med Edu Res 2015;49(1):5-9.

Source of support: Nil

Conflict of interest: None

\footnotetext{
${ }^{1}$ Consultant Spine Surgeon, ${ }^{2-5}$ Spine Fellow

${ }^{6}$ Consultant Neuroanesthetist

${ }^{1-6}$ Department of Neurosciences, Park Clinic, Kolkata West Bengal, India
}

Corresponding Author: Agnivesh Tikoo, Spine Fellow, Department of Neurosciences, Park Clinic, Kolkata, West Bengal, India Phone: 8879005175, e-mail: agniveshtikoo@gmail.com

\section{INTRODUCTION}

Adequate treatment of spinal lesions requires formulation of diagnosis-best achieved by a tissue biopsy when all attempts at diagnosis fail by noninvasive methods. More often these lesions represent the disease process that originated elsewhere. ${ }^{1}$ Open biopsy is considered to be the gold standard with $98 \%$ accuracy. ${ }^{2} \mathrm{Craig}^{3}$ in 1956 developed the technique and needle for core biopsy (open) of the vertebrae, by a paravertebral approach, but it was associated with significant hemorrhage and few incidences of root damage. Other complications of open biopsy that have been quoted include skin, bone, and soft-tissue problems (prevalence 17\%), the risk of a diagnostic error (prevalence $18 \%$ ), and the risk of missing a small lesion. ${ }^{4}$ However, percutaneous technique can avoid the morbidity of the procedure giving similar results. Stringham et $\mathrm{al}^{5}$ showed percutaneous biopsy to be more cost effective and less invasive than the open biopsy. Percutaneous CT-guided fine needle biopsy is technically difficult in intraosseous lesions leading to frequent inconclusive results and hence the necessity of wide bore needle biopsy. The procedure can be done under local anesthesia with or without sedation. Ottolenghi ${ }^{6}$ in 1955 described results of the large series of percutaneous needle biopsy $(n=1061)$ which included 204 vertebral bodies and quoted an overall positivity rate of $84.35 \%$ for the procedure. Fyfe et $\mathrm{al}^{7}$ showed that needle biopsy with core diameter more than $2 \mathrm{~mm}$ had high degree of diagnostic accuracy.

\section{MATERIALS AND METHODS}

Retrospective analysis of data of 26 patients who underwent a percutaneous transpedicular biopsy at our hospital was done. All patients had a bony lesion in a vertebra (thoracic, lumbar, saccrum) without a soft tissue component around the bone and without any neurodeficit. Those cases which had associated soft tissue component/abscess/collection were excluded because they were considered more suitable for CT-guided aspiration. The cases with any impending instability were also excluded 26 cases ( 15 females and 11 males) were included in the study. Mean age was 58.8 years. All patients had undergone preoperative skiagrams, CT scan and MRI. Additional investigations like bone scan, 
blood tests (CRP/protein electrophoresis, etc.) were done whenever indicated. The most common level involved was L1 (n = 5) (Graph 1).

Transpedicular biopsy was used as a diagnostic procedure in all cases where the routine screening tests (biochemical/clinical/radiological) were inconclusive for any definitive diagnosis. There were three cases which also had adjacent level changes on MRI/collapses on skiagram. In these cases, the vertebral level which showed maximum change on MRI and/or CT was considered.

All cases were done by infiltrating xylocaine $2 \%$ into skin, subcutaneous tissue and periosteum. Intravenous sedation with propofol and fentanyl was used in 11 patients in addition to xylocaine.

Patients were positioned prone on the table with hips and knees in 30 to $40^{\circ}$ flexion over bolsters. The side of insertion of the needle was guided by the location of pathology. Level was identified under fluoroscopy and marked. Skin was incised with an eleven number skin knife. Eleven gauge disposable Jamshidi (vertebroplasty) needle was used. The needle was introduced into the pedicle under fluoroscopic guidance (Fig. 1) and was directed to the area of pathology if visible under fluoroscopy, otherwise we tried to advance the needle to that part of vertebral body that contained the lesion as depicted by preoperative investigation (CT, MRI). The stylet was withdrawn from needle once the edge of the lesion or pedicle-body junction was reached and the needle was advanced further. The needle was withdrawn and the biopsy obtained was inspected macroscopically with respect to the nature and the amount of tissue collected. This procedure was repeated 2 to 3 times. If the biopsy collected was not satisfactory, then additional instruments like curette and pituitary forceps can be introduced through the same incision to get additional

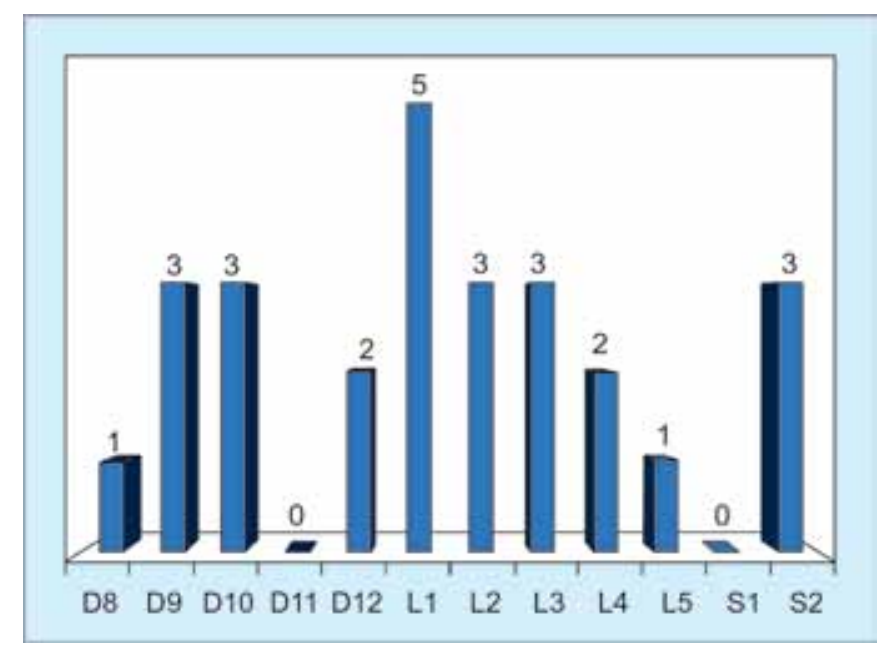

Graph 1: Distribution of vertebral levels biopsy material. We used this method in three of our patients (Fig. 2). The biopsy obtained was sent for histopathology. All patients were discharged the next day except one case which developed transient neurodeficit.

\section{RESULTS}

The plain skiagrams were negative in 4 out of 26 cases (15.3\%). These skiagrams did not show any osteolytic/ osteoblastic lesion nor did they show any loss of height or trabeculations. Five cases (19.2\%) showed loss of trabeculae only. Out of the remaining 17 cases, 10 cases (38.4\%) showed mild wedging (loss of upto $25 \%$ height anteriorly) which could be appreciated better by comparing them with adjacent vertebrae. Seven cases (26.9\%) showed significant wedging ( $>25 \%$ loss of height).

MRI findings were uniformly present as hypointensity in T1 and hyperintense signal in T2 and STIR in all the cases $(100 \%)$. CT scan was more descriptive than skiagram/MRI with respect to localization of the main focus of lesion and helped to plan the track of biopsy.

The center of the lesion/lesion was present in anterior/center body in 23 cases (88.4\%). It was present in
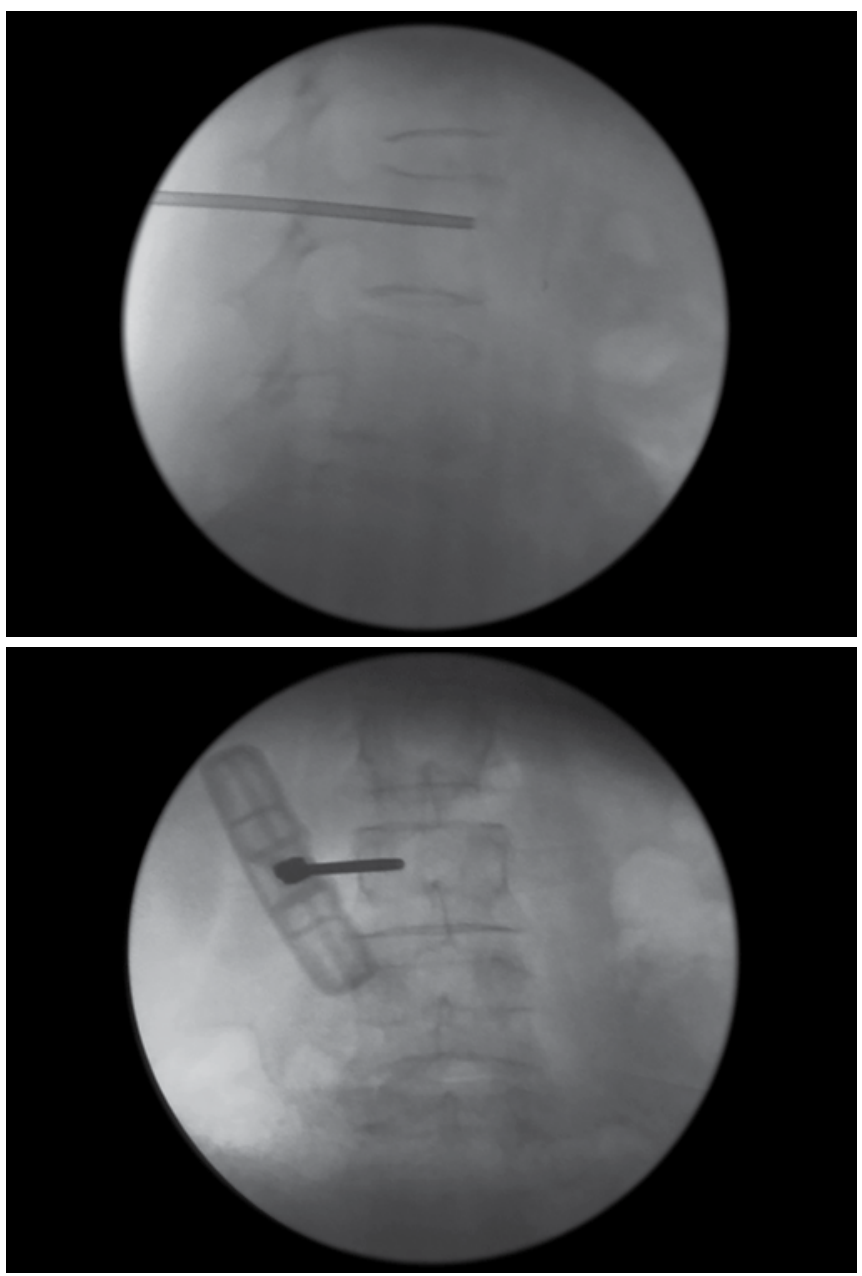

Fig. 1: Checking position of 11 gauge disposable vertebroplasty needle in two planes 
pedicle body junction in two cases $(7.6 \%)$, anterior part of pedicle in 1 cases (3.8\%). Based on the location, the appropriate area from which biopsy needs to be taken was planned preoperatively and needle was progressed to same area under fluoroscopic control.

The biopsy material obtained was considered adequate if there was a track of tissue containing normal bone and fleshy mass (pathological bone/tissue). If this cylindrical tissue was not obtained, attempts were made to gather such tissue by altering the direction of the needle. We were able to attain tissue in first 2 to 3 attempts in 21 cases $(75 \%), 3(10.7 \%)$ cases required needles to be re-inserted more than 3 times. Attempts to insert needle was abandoned if no satisfactory tissue was obtained even after 4 th attempt. In that case, we used additional instruments (curette, disk forceps) through the same incision (Fig. 2). We used additional instruments in 4 cases $(14.2 \%)$.

- Overview: Positive diagnosis was achieved in 23 out of 26, i.e. $88.4 \%$ of cases (adequacy). Out of 26 , there were 13 cases of malignancies (50\%), 8 cases of tuberculosis (30.7\%), 2 cases of osteoporosis (7.6\%) and biopsy was inconclusive in $3(11.5 \%)$ cases. Of the 13 malignancies,
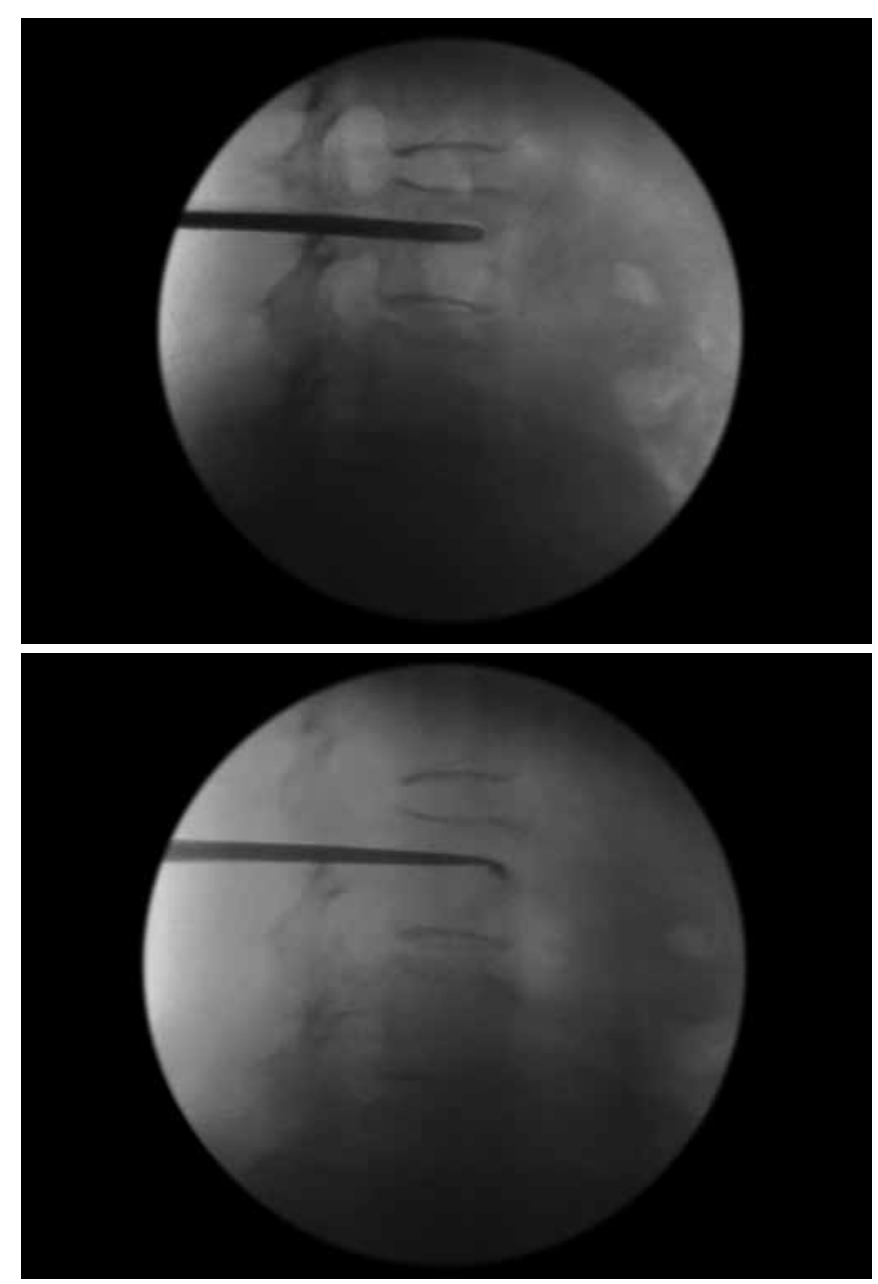

Fig. 2: Using disk forceps and curette for obtaining additional tissue
7 cases were of metastasis (53.8\%), 5 cases of plasmocytoma (38.4\%) and 1 case of lymphoma (7.6\%). The 7 cases of metastasis histologically were secondary carcinomas in $5(71.4 \%)$ and, papillary carcinoma and adenocarcinoma in one (14.2\%) each. We were able to obtain diagnosis in all the patients in which additional instrumentation was used. Also, in cases which had multiple collapses, the idea of obtaining a biopsy from the lesion which showed maximum changes on MRI was also successful in all the cases $(n=3)$.

- Inconclusive results: Of the 3 inconclusive results one patient improved with a trial of ATD and that was continued for 12 months. At the end of 12 months patient had good healing radiologically with remission of clinical symptoms and inflammatory markers (total and differential leukocyte counts, ESR and CRP) completely. One patient underwent open biopsy and turned out to be secondary carcinoma. One patient refused to undergo any further procedure and was lost to follow-up.

- Complications: No major complications were encountered. Nine patients (34.6\%) had mild postoperative pain which was managed with analgesics. One patient who had a lesion at D8 showed transient neurological deficit in form of monoparesis of the lower limb of the same side from which the biopsy was being taken. The patient was given injection methylprednisolone $1 \mathrm{gm}$ postoperative and was taken for CT and MRI which did not show any breach/fracture of medial cortex of the pedicle or any cord injury. Patient was discharged with improving monoparesis on 6th day which recovered completely in 6 weeks. There were no cases of hematoma formation or secondary infection. The patient's histopathology was suggestive of tuberculosis. Patient was accordingly put on ATT for 1 year and improved completely.

\section{DISCUSSION}

More often than not many diseases present only by a vertebral lesion with all attempts at diagnosis failing by routine screening tests. In such cases transpedicular biopsy is a potentially viable procedure providing affective diagnosis. It can be done as a day care procedure under local anesthesia (with or without sedation) with reasonable safety.

Skiagrams were reported normal in 4 cases with additional 5 cases having only trabecular loss. These cases were only diagnosed when MRI was used as next investigation. Therefore, high suspicion is needed whenever a patient presents with backpain of long duration with night pains otherwise with plain skiagrams may be false negative in $15.3 \%$ of the cases and findings may 
be missed in another $19.2 \%$ of patients if the consulting physician is not careful.

Kattapuram et $\mathrm{al}^{8}$ showed that there is a slight (nonsignificant) increase in the accuracy of biopsies performed with use of large-bore needles (needles with a larger inner diameter) as compared with fine needles. Fyfe et al in a cadaveric study found that diagnostic yield increased from 50 to $90 \%$ while using needled with core diameter of $2 \mathrm{~mm}$ which was attributed to the crush effect of the smaller diameter needles on the bone tissue. ${ }^{7}$ Logan et al ${ }^{9}$ found increased accuracy in association with core biopsy as compared with fineneedle sampling. The patients with sclerotic lesions cannot undergo biopsy with fine needles. ${ }^{10,11}$ We used 11 gauge disposable Jamshidi (Vertebroplasty) needle was used which has an internal diameter of $2.3 \mathrm{~mm}$ and an outer diameter of $3 \mathrm{~mm}$. However, if it was found that the biopsy obtained by this needle was insufficient, use of additional instrumentation was considered and proved beneficial. In our series in we were able to obtain diagnosis in all 4 cases in which additional instrumentation was used. The use of additional instrumentation in our series was not associated with any increase in complication rate or any postoperative pain. In case of multiple lesions $(n=3)$, the level which showed maximum changes on MRI was considered for biopsy. In these, we were also were able to get a positive diagnosis in all of the cases which were osteoporotic collapse, tuberculosis and NHL. In those cases in which we were unable to make a histological diagnosis, all had bright signals on $\mathrm{T} 2$ with minimal changes on CT. The cases where both CT and MRI were considered together were more effective in giving a positive histological diagnosis rather than MRI alone.

Paraspinal approach has been classically described as a routine method of obtaining vertebral biopsy., 8,10,12-14 The complications associated with paraspinal approach is more pronounced in thoracic spine with incidences of pneumothorax in 4 to $11 \%$ of patients and incidences of radicular pain, nerve root injury and hematoma formation. ${ }^{15}$ Moreover, intact lateral cortex cannot be penetrated by this approach. Many studies have shown that transpedicular biopsy if done with caution obviates many of these complications. ${ }^{14-16}$ Jelinek et $\mathrm{al}^{15}$ in his study on 32 patients concluded that transpedicular biopsy of deep vertebral body lesions using a bone biopsy needle under computed tomography or fluoroscopy guidance can be performed safely and efficaciously as an outpatient procedure. The bleeding through the needle, which may sometimes occur, can be easily controlled by gelfoam packing. ${ }^{17,18}$
CT guided FNAC is more suitable for soft tissue component of the vertebral lesion; however it is difficult to get aspirate from the bone. Even if we are able to get aspirate or biopsy with a smaller needle from the vertebra, it tends to produce more smears rather than true biopsy and due to crush effect, obscures the histological diagnosis. Large diameter needles tend to produce artefacts on CT scan and tend to obscure the anatomy. Nourbaksh et $\mathrm{al}^{2}$ in his meta-analysis found that studies done with CT guidance showed slightly higher rates for adequacy $(92.6 \%$ compared with $90.1 \%$, $\mathrm{p}=0.40)$ and accuracy $(90.2 \%$ compared with $88.1 \%, \mathrm{p}=$ 0.59 ) in comparison with fluoroscopy, which although were statistically insignificant.

Adequacy has been defined as percentage of those biopsies with which a diagnosis can be made and hence includes true positives and true negatives (normal biopsy). ${ }^{2}$ Accuracy is the percentage of biopsy reports that are confirmed either after surgery, on the basis of an open biopsy, in response to treatment, or at the time of follow-up; thus, accuracy is equal to the true-positive rate plus the true-negative rate. Previous studies have showed a high overall accuracy for biopsy of primary and secondary tumors, accuracy between 82 and $94 \% .{ }^{15}$ The adequacy and accuracy achieved in our study was 88.4 and $100 \%$ respectively.

Transpedicular fluoroscopic guided biopsy can give good results and may avoid wrong treatment on empirical basis or delay in treatment. All the cases which were subsequently diagnosed as tuberculosis with histopathology of tuberculosis which did not have any associated paravertebral abscess. Even biochemical markers were not conclusive in 4 out of $8(50 \%)$ cases. These cases were diagnosed only by biopsy and all showed adequate resolution (clinical/radiological/biochemical) at the end of 1 year. Likewise, out of the malignancy cases, 3 out of 14 cases had raised biochemical markers (ESR and CRP) with positive history of weight loss and night sweats but in biopsy they turned out to be metastasis in 2 and lymphoma in one.

\section{CONCLUSION}

Percutaneous biopsy under fluoroscopic guidance by transpedicular approach is safe and gives high adequacy $(88.4 \%)$ without significant complications that are associated with open and paraspinal techniques. It can safely be performed as an out patient procedure. It also reduces the risk of wrong diagnosis and prevents delay in appropriate treatment. It therefore is a very good and effective procedure for occult vertebral lesions presenting without any soft tissue component. In patients with 
multiple collapses/signal intensity changes on MRI, the level with maximum changes on MRI and CT should be considered for biopsy.

\section{REFERENCES}

1. Odendaal T, Lemmer LB. The value of percutaneous trephine biopsy in the diagnosis of lesions of the vertebral column. S Afr Med J 1991 Jan 5;79(1):21-23.

2. Nourbakhsh A, Grady JJ, Garges KJ. Percutaneous spine biopsy: a meta-analysis. J Bone Joint Surg Am 2008 Aug; 90(8):1722-1725.

3. Craig FS. Vertebral body biopsy. J Bone Joint Surg Am 1956 Jan;38A(1):93-102.

4. Mankin HJ, Lange TA, Spanier SS. The hazards of biopsy in patients with malignant primary bone and soft-tissue tumors. J Bone Joint Surg Am 1982 Oct;64(8):1121-1127.

5. Stringham DR, Hadjipavlou A, Dzioba RB, Lander P. Percutaneous transpedicular biopsy of the spine. Spine (Phila Pa 1976) 1994 Sep 1;19(17):1985-1991.

6. Ottolenghi CE. Diagnosis of orthopaedic lesions by aspiration biopsy; results of 1,061 punctures. J Bone Joint Surg Am 1955 Jun;37A(3):443-464.

7. Fyfe IS, Henry APJ, Mulholland RC. Closed vertebral biopsy. J Bone Joint Surg Br 1983 March;65(2):140-143.

8. Kattapuram SV, Khurana JS, Rosenthal DI. Percutaneous needle biopsy of the spine. Spine (Phila Pa 1976) 1992 May;17(5): 561-564.

9. Logan PM, Connell DG, O'Connell JX, Munk PL, Janzen DL. Image-guided percutaneous biopsy of musculoskeletal tumors: an algorithm for selection of specific biopsy techniques. AJR Am J Roentgenol 1996 Jan;166(1):137-141.

10. Ghelman B, Lospinuso MF, Levine DB, O'Leary PF, Burke SW. Percutaneous computed-tomography-guided biopsy of the thoracic and lumbar spine. Spine (Phila Pa 1976) 1991 July; 16(7):736-739.

11. Lis E, Bilsky MH, Pisinski L, Boland P, Healey JH, O'Malley B, Krol G. Percutaneous CT-guided biopsy of osseous lesion of the spine in patients with known or suspected malignancy. AJNR Am J Neuroradiol 2004 Oct;25(9):1583-1588.

12. Adapon BD, Legada BD, Lim EVA, Silao JV Jr, Dalmacio-Cruz A. CT-guided closed biopsy of the spine. J Comput Assist Tomogr 1981 Feb;5(1):73-78.

13. Bender CE, Berquist TH, Wold LE. Imaging-assisted percutaneous biopsy of the thoracic spine. Mayo Clin Proc 1986 Dec;61(12):942-950.

14. Stoker DJ, Dissin CM. Percutaneous vertebral biopsy: a review of 135 cases. Clin Radiol 1985 Nov;36(6):569-577.

15. Jelinek JS, Kransdorf MJ, Gray R, Aboulafia AJ, Malawer MM. Percutaneous transpedicular biopsy of vertebral body lesions. Spine (Phila Pa 1976) 1996 Sep 1;21(17):2035-2040.

16. Renfrew DL, Whitten CG, Wiese JA, El-Khoury GY, Harris KG. CT guided percutaneous transpedicular biopsy of the spine. Radiology 1991 Aug;180(2):574-576.

17. YaffeD,Greenberg G, Leitner J,Gipstein R, Shapiro M, BacharGN. CT-guided percutaneous biopsy of thoracic and lumbar spine: a new coaxial technique. Am J Neuroradiol 2003 Nov-Dec; 24(10):2111-2113.

18. Christodoulou A, Samoladas E, Givissis P, Pournaras I. Pulsatile bleeding during closed vertebral biopsy. Acta Orthop Belg 2007 Dec;73(6):778-779. 